\title{
OPEN Mammographic tumour appearance is related to clinicopathological factors and surrogate molecular breast cancer subtype
}

Li Sturesdotter $\mathbb{1}^{1,2 \rrbracket}$, Malte Sandsveden $\mathbb{1}^{3,4}$, Kristin Johnson $\mathbb{1}^{1,2}$, Anna-Maria Larsson $\mathbb{1}^{5,6}$, Sophia Zackrisson $\mathbb{1}^{1,2}$ \& Hanna Sartor ${ }^{101,2}$

Mammographic tumour appearance may provide prognostic useful information. For example, spiculation indicates invasiveness, but also better survival compared to tumours with other appearances. We aimed to study the relationship between mammographic tumour appearance and established clinicopathological factors, including surrogate molecular breast cancer subtypes, in the large Malmö Diet and Cancer Study. A total of 1116 women with invasive breast cancer, diagnosed between 1991 and 2014, were included. Mammographic tumour appearance in relation to status for oestrogen receptor (ER), progesterone receptor (PR), human epidermal growth factor receptor 2, histological grade, Ki67 and molecular subtype was analysed using various regression models. All models were adjusted for relevant confounders, including breast density, which can affect mammographic appearance. The results consistently showed that spiculated tumours are indicative of favourable characteristics, as they are more likely to be ER and PR positive, and more often exhibit lower histological grade and lower Ki67 expression. Furthermore, spiculated tumours tend to be of luminal A-like subtype, which is associated with a good prognosis. The establishment of associations between mammographic tumour appearance and clinicopathological factors may aid in characterizing breast cancer at an earlier stage. This could contribute to more individualized breast cancer treatment in the future.

Mammography plays a fundamental role in breast cancer screening and diagnosis ${ }^{1}$. The images can provide crucial information, although their full potential has not yet been utilized in clinical practice. The mammographic appearance of breast lesions, both benign and malignant, corresponds to histopathological diagnoses, which can be predicted with varying certainty. For example, a spiculated (or stellate) lesion has a high positive predictive value for malignancy, often around $90 \%^{2}$, whereas a mass with a circumscribed contour most often indicates a benign lesion (e.g. fibroadenoma, cyst or hamartoma) ${ }^{3}$. However, a circumscribed mass can also be an invasive carcinoma without surrounding stromal reaction ${ }^{3}$, making diagnosis based on imaging alone unreliable. Several previous studies have been performed on the relation between the mammographic appearance of malignant tumours and diagnosis and survival. For example, tumours with casting-type calcifications are associated with a worse prognosis ${ }^{4}$. On the other hand, spiculation indicates invasiveness ${ }^{5}$, but at the same time a better survival rate than in those breast tumours with other mammographic appearances ${ }^{6}$. Breast density is also known to affect mammographic appearance ${ }^{7}$. Knowledge on clinicopathological factors such as tumour size, histological type, hormonal receptor status and axillary lymph node involvement is essential in breast cancer staging and the prediction of prognosis ${ }^{8}$. Our research group has previously studied mammographic tumour appearance in relation

\footnotetext{
${ }^{1}$ Department of Translational Medicine, Diagnostic Radiology, Lund University, Lund, Sweden. ${ }^{2}$ Department of Medical Imaging and Physiology, Skåne University Hospital, Lund/Malmö, Sweden. ${ }^{3}$ Department of Clinical Sciences Malmö, Surgery, Lund University, Lund, Sweden. ${ }^{4}$ Department of Surgery, Skåne University Hospital, Malmö, Sweden. ${ }^{5}$ Department of Clinical Sciences Lund, Division of Oncology, Lund University, Lund, Sweden. ${ }^{6}$ Department of Hematology, Oncology and Radiation Physics, Skåne University Hospital, Lund, Sweden. ${ }^{凶}$ email: li.sturesdotter@med.lu.se
} 
to invasiveness, tumour size and axillary lymph node involvement within a smaller subset of the same cohort used in this study, and found an association between spiculation and invasiveness, regardless of breast density ${ }^{5}$.

In addition to clinicopathological factors, surrogate molecular subtypes aid in the prediction of prognosis and the choice of appropriate treatment ${ }^{8}$. It has been suggested in the literature that the presence of calcifications on mammography is more common in the human epidermal growth factor receptor 2 amplified (HER2+) subtype ${ }^{9,10}$, while triple-negative breast cancer (TNBC) is more likely to present as a mass ${ }^{10}$. Few previous studies have, however, investigated the combination of the information available from mammographic appearance, clinicopathological factors, including molecular subtypes, and breast density. The aim of the present study was thus to investigate the relationship between mammographic tumour appearance and clinicopathological factors, including surrogate molecular subtypes, in the large cohort of incident breast cancer cases within the Malmö Diet and Cancer Study.

\section{Methods and material}

Study population. Data were collected from the Malmö Diet and Cancer Study (MDCS), a large population-based prospective cohort study, in which 28,098 individuals were enrolled from 1991 to 1996, of which 17,035 were women ${ }^{11}$. Inhabitants aged 44-74 years were recruited from the city of Malmö in the south of Sweden. The original intent of the study was to investigate possible associations between diet and cancer. At the baseline examination, anthropometric variables (blood pressure, height, weight, lean body mass and body fat mass) and blood samples were collected, and a comprehensive questionnaire was filled in ${ }^{12}$. The MDCS is updated regularly with information on new cancer cases through data collection from national registers: the Swedish Cause of Death Register, the Swedish Cancer Register, and the Regional Tumour Register for Southern Sweden ${ }^{5}$. Women with prevalent breast cancer at baseline or a history of breast cancer $(n=572)$ were excluded. A total of 1242 women in the cohort were diagnosed with incident breast cancer between 1991 and 2014. After the exclusion of carcinoma in situ $(n=105)$ and bilateral breast tumours $(n=21), 1116$ women with invasive breast cancer remained eligible for the present study. Informed consent was obtained at the baseline examination. The Ethics Committee at Lund University approved this study (Official Records Nos. 652/2005 and 166/2007). The study was carried out in accordance with the declaration of Helsinki.

Mammography. A protocol was set up to extract information from the original mammography report at the time of cancer diagnosis. This protocol had several variables, including mammographic tumour appearance, breast density and mode of cancer detection ${ }^{5}$. All screening mammograms were double read by two breast radiologists, but the diagnostic imaging during follow-up assessment was performed by one breast radiologist. Mammograms of clinically detected cancers were single read by one breast radiologist. When any of the information was lacking in the original mammography report, the mammograms were re-read by an experienced breast radiologist (SZ and/or HS).

Mammographic tumour appearance. Information on the most dominant mammographic appearance of the tumour was obtained retrospectively. It should be noted that more than one appearance could have been readily visible and described in the original report, but only the most dominant appearance was recorded in the study protocol. Tumours were classified into the following comprehensive categories, based on the work by Luck et al. ${ }^{13}$ : well-defined mass, partly ill-defined mass, ill-defined/diffuse mass, spiculated mass, comedo-type microcalcifications, non-specific calcifications, architectural distortion and asymmetrical density. For statistical analysis, these categories were converted into five larger categories: distinct mass (including well-defined and partly ill-defined tumours), ill-defined mass, spiculated mass, calcifications (including comedo-type and nonspecific calcifications) and tissue abnormality (including the less frequent features architectural distortion and asymmetrical density). Some of the appearances are illustrated in Fig. 1.

Breast density. In the clinical setting at the Department of Breast Radiology in Malmö, breast density is divided into three groups: fat involuted, moderately dense and dense (Fig. 2). These three groups were employed in the present study. Fat involuted corresponds to Breast Imaging Reporting and Data System (BI-RADS) $4^{14}$ density score 1, moderately dense to BI-RADS 4 density score 2-3, and dense to BI-RADS 4 density score 4.

Mode of cancer detection. Method of cancer detection was divided into screening-detected or clinically detected, i.e. either detection via the breast cancer screening programme that started in Malmö in 1990, or detected clinically, due to a lump in the breast or other symptoms that caused the woman to seek medical attention. Thus, clinically detected tumours also include interval cancers, i.e. cancers diagnosed between screening episodes.

Clinicopathological tumour factors. Information regarding clinicopathological factors was extracted from medical records and tissue microarray (TMA) evaluation. One TMA was constructed for breast cancers diagnosed between 1991 and 2004, and another one for breast cancers diagnosed between 2005 and 2007. For each tumour, two cores (0.6 mm in size 1991-2004 and $1.0 \mathrm{~mm}$ in size 2005-2007) from different representative areas of the tumour were collected for TMA construction. The details concerning the TMA construction have been described previously ${ }^{15}$.

Hormone receptors. Oestrogen receptor (ER) and progesterone receptor (PR) expression, based on immunohistochemical (IHC) staining, was extracted from TMA evaluation for the period 1991-2004, and from medi- 

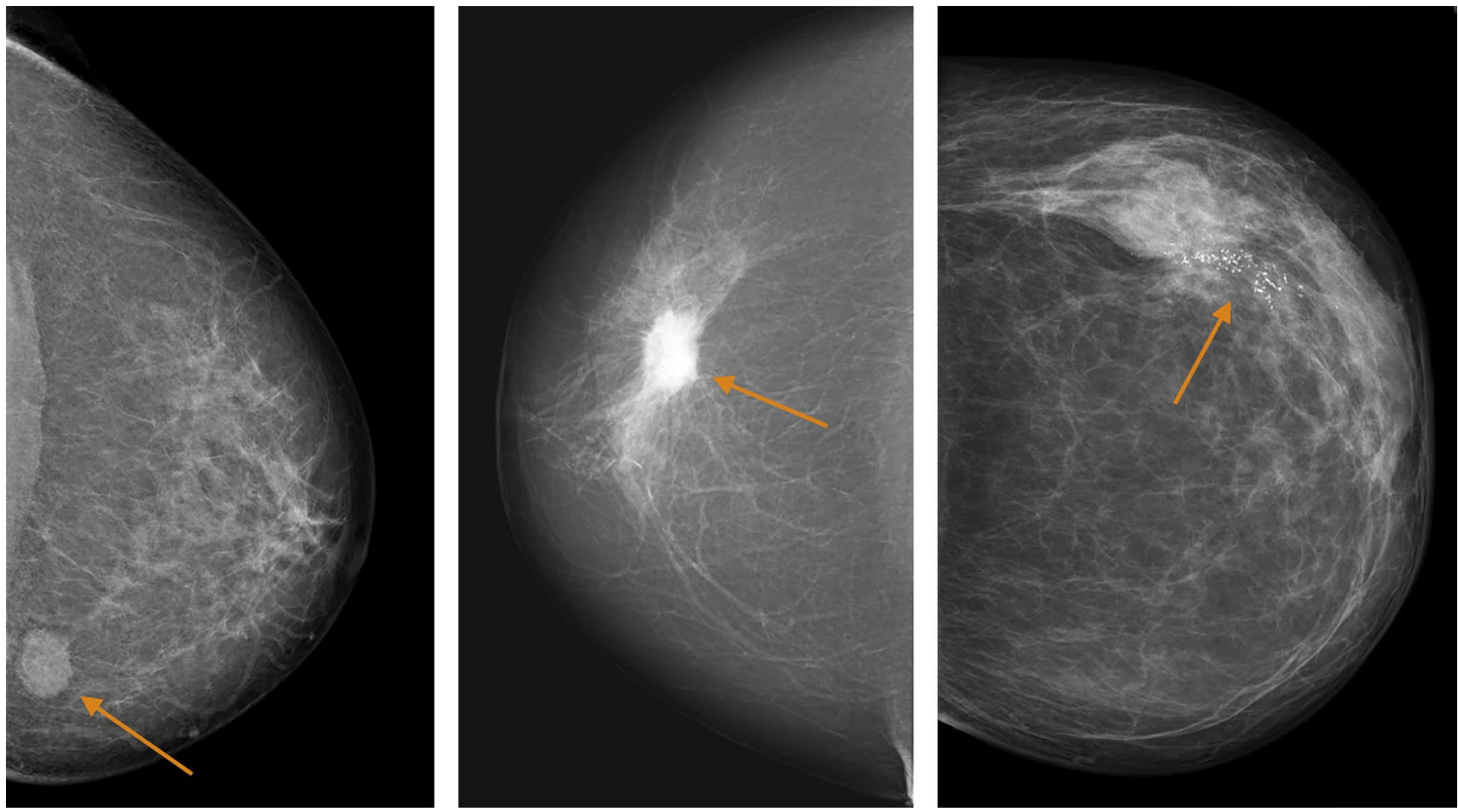

Figure 1. Mammographic tumour appearances. From left to right: distinct mass in the left breast of an 80-yearold woman, moderately dense breast. Spiculated mass in the right breast of 79-year-old woman, fat involuted breast. Calcifications in the left breast of a 77-year-old woman, moderately dense breast. All images acquired in the craniocaudal projection.
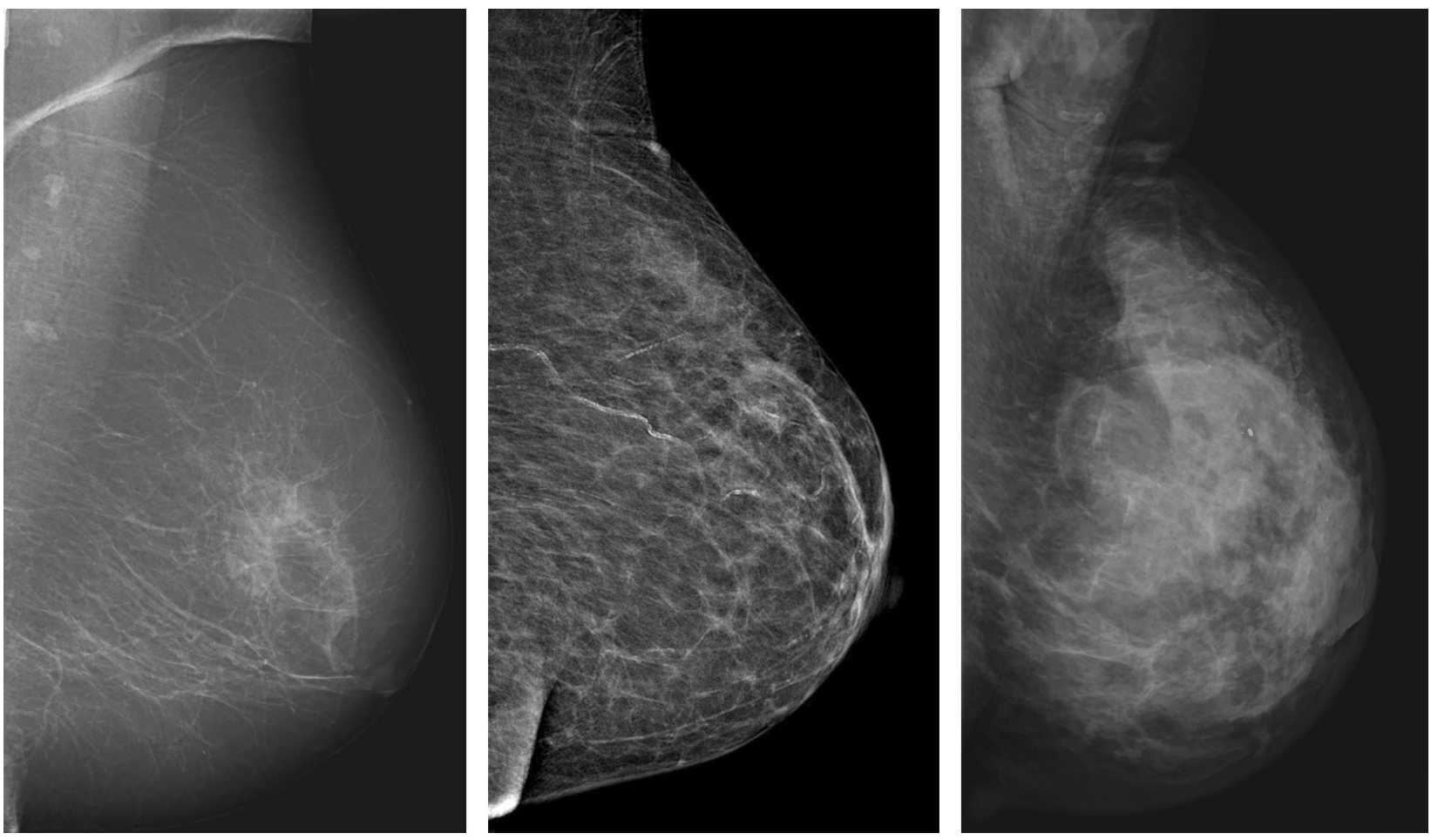

Figure 2. Breast density. From left to right: fat involuted breast. Moderately dense breast. Dense breast. All images acquired in the mediolateral oblique projection. 
cal records for the period 2005-2014. IHC staining of 10\% positive cells or less was considered negative, while values above $10 \%$ were interpreted as positive, in accordance with local clinical practice.

HER2. Information on HER2 status was retrieved from TMA evaluation from 1991 to 2007, and from medical records from 2008 to 2014. From 1991 to 2004, HER2 status was based solely on the IHC HercepTest ${ }^{16}$. Values of 0 and $1+$ were considered HER2 non-amplified (HER2-), and 3+ amplified (HER2+), while 2+ was entered as missing. In situ hybridization analysis was available from 2005 and onwards, and category $2+$ according to the HercepTest were from 2005 and onwards either categorized as amplified or non-amplified if conclusive in situ hybridization analysis had been performed, otherwise as missing, as described by Elebro et al. ${ }^{15}$.

Histological grade and tumour type. Breast cancers diagnosed from 1991 to 2004 were included in the first TMA and the histological grade (also Nottingham grade or Elston grade ${ }^{17}$ ) and histological type according to the World Health Organization classification was re-assessed by an experienced breast pathologist. For the period 2005-2014, this information was extracted from medical records ${ }^{18}$.

Ki67. Information on IHC proliferation marker Ki67 expression was collected during three time periods, from TMA assessment 1991-2004 and 2005-2007, and from medical records 2008-2014. Estimates of Ki67 expression can vary due to inter- and intra-observer variability, and due to variability in staining between pathology laboratories ${ }^{19,20}$. Ki67 status in the MDCS cohort varies during the long follow-up period of 13 years. Thus, using the same cut-off values for all three time periods could be misleading. To overcome this risk in the MDCS cohort, the values were divided into three equally sized groups for each time period ${ }^{18}$. The group with the lowest scores is denoted "low", middle scores "intermediate", and highest scores "high".

Tumour size. Data on tumour size were collected from medical records during the entire follow-up period and were based on histopathological measurements. In multifocal breast cancer, the largest focus was used.

Surrogate molecular subtypes. The tumours were subsequently classified into surrogate molecular breast cancer subtypes in a modified St. Gallen $2013^{21}$ manner to facilitate the identification of different prognoses and characteristics. The mode of categorization was based on the local agreement of the southern Swedish breast cancer group, using the following subtypes: luminal A-like, luminal B-like, HER2+subtype and TNBC ${ }^{22}$. All grade 1, ER positive $(\mathrm{ER}+)$ tumours were categorized as luminal A-like, regardless of Ki67 and PR status. Grade 2 $\mathrm{ER}+$ tumours with low Ki67 expression, and grade 2 with intermediate Ki67 expression combined with PR positive $(\mathrm{PR}+)$ status were also classified as luminal A-like. All grade $3 \mathrm{ER}+$ tumours were considered to be luminal B-like, regardless of Ki67 and PR status. Grade 2 ER+tumours with high Ki67 expression or intermediate Ki67 and PR- were classified as luminal B-like. All HER2+ tumours were considered HER2+ subtype, regardless of grade and hormone receptor status. ER-, PR- and HER2- tumours were considered TNBC.

Statistical methods. Descriptive statistics, logistic regression, ordinal regression and multinomial logistic regression were carried out. The relation between mammographic appearance and ER, PR, and HER2 status was analysed with logistic regression, generating odds ratios (OR) and 95\% confidence intervals (CI). To study the distribution of tumour appearance within the three categories of histological grade and Ki67, ordinal regression models were used, generating OR and 95\% CI. The proportional odds assumption was assessed for each model, and if it holds, the OR:s can be interpreted as odds of being category 3 compared to the combined odds of being category 1 and 2. Potential associations between mammographic tumour appearance and surrogate molecular breast cancer subtypes were assessed through multinomial logistic regression, where distinct mass was set as a reference for the tumour appearances, and luminal A-like as a reference for the subtypes, yielding relative risk ratios (RRR) with $95 \% \mathrm{CI}$.

All statistical calculations were adjusted for breast density, mode of cancer detection, age at diagnosis and tumour size. Breast density was divided into three groups, and mode of detection into screening or clinical, as described in the mammography section above. Age at diagnosis was divided into quartiles. Tumour size was dichotomised into $\leq 20 \mathrm{~mm}$ and $>20 \mathrm{~mm}$. In all the analyses, a p-value of less than 0.05 was considered statistically significant. Statistical analyses were performed with Stata version SE 14.2.

\section{Results}

Table 1 provides information on the study population, including patient and tumour characteristics, in relation to mammographic appearance.

Tumour appearance in relation to clinicopathological factors. Ill-defined tumours, spiculated tumours and tumours presenting as tissue abnormality were more likely to be ER+ than ER-, as compared to a distinct mass, according to logistic regression analysis, with an adjusted $\mathrm{OR}\left(\mathrm{OR}_{\mathrm{adj}}\right)$ of 2.0 (CI 1.1-3.6), 6.0 (CI 3.2-11.2) and 4.4 (CI 1.0-19.6), respectively (Table 2). Furthermore, spiculated tumours were more likely to be $\mathrm{PR}+$ than PR- compared to a distinct mass, with an $\mathrm{OR}_{\text {adj }}$ of 1.7 (CI 1.2-2.5) (Table 2). We found no statistical evidence for an association between mammographic appearance and HER2 status (Table 2). However, ill-defined masses and tumours presenting as calcifications were slightly more often HER2+ than were the other tumour features in terms of distribution. According to ordinal regression analysis, the odds of spiculated tumours, compared to distinct masses, to be histological grade 3, compared to the combined categories of grade 1 and 2, were $50 \%$ lower, $\mathrm{OR}_{\text {adj }} 0.5$ (CI 0.4-0.7), given that all the other variables in the model were kept constant (Table 3). 


\begin{tabular}{|c|c|c|c|c|c|c|}
\hline & Distinct mass & Ill-defined mass & Spiculated & Calcifications & Tissue abnormality & Total \\
\hline Age at diagnosis, median (range) & $67(48-89)$ & $66(45-91)$ & $66(49-91)$ & $64(48-83)$ & $67(48-89)$ & $66(45-91)$ \\
\hline \multicolumn{7}{|c|}{ Breast density } \\
\hline Fat involuted & $57(21.9)$ & $30(15.4)$ & $83(20.2)$ & $4(5.1)$ & $6(15.4)$ & $180(18.3)$ \\
\hline Moderately dense & $139(53.5)$ & $96(49.2)$ & $213(52.0)$ & $30(38.5)$ & $12(30.7)$ & $490(49.9)$ \\
\hline Dense & $64(24.6)$ & $69(35.4)$ & $114(27.8)$ & $44(56.4)$ & $21(53.9)$ & $312(31.8)$ \\
\hline Missing & 6 & 8 & 6 & 5 & 2 & 27 \\
\hline \multicolumn{7}{|l|}{ Mode of detection } \\
\hline Screening detection & $114(43.0)$ & $88(43.4)$ & $253(60.8)$ & $62(74.7)$ & $16(39.0)$ & $533(52.9)$ \\
\hline Clinical detection & $151(57.0)$ & $115(56.7)$ & $163(39.2)$ & $21(25.3)$ & $25(61.0)$ & $475(47.1)$ \\
\hline Missing & 1 & 0 & 0 & 0 & 0 & 1 \\
\hline \multicolumn{7}{|l|}{ Tumour size (mm) } \\
\hline$\leq 20$ & $182(73.7)$ & $108(55.7)$ & $308(75.3)$ & $69(85.2)$ & $15(45.5)$ & $682(70.8)$ \\
\hline$>20$ & $65(26.3)$ & $86(44.3)$ & $101(24.7)$ & $12(14.8)$ & $18(54.5)$ & $282(29.2)$ \\
\hline Missing & 19 & 9 & 7 & 2 & 8 & 45 \\
\hline \multicolumn{7}{|l|}{ Histological grade } \\
\hline 1 & $56(24.0)$ & 34 (17.7) & $127(31.4)$ & $22(29.3)$ & $11(34.4)$ & $250(26.7)$ \\
\hline 2 & $91(39.1)$ & 95 (49.5) & $210(51.9)$ & $32(42.7)$ & $14(43.7)$ & $442(47.2)$ \\
\hline 3 & $86(36.9)$ & $63(32.8)$ & $68(16.7)$ & $21(28.0)$ & $7(21.9)$ & $245(26.1)$ \\
\hline Missing & 33 & 11 & 11 & 8 & 9 & 72 \\
\hline \multicolumn{7}{|l|}{ Histological type } \\
\hline Invasive ductal cancer & $183(76.6)$ & $130(67.0)$ & $272(67.3)$ & $68(85.0)$ & $17(53.1)$ & $670(70.6)$ \\
\hline Invasive lobular cancer & $12(5.0)$ & $56(28.9)$ & $92(22.8)$ & $7(8.7)$ & $13(40.6)$ & $180(19.0)$ \\
\hline Invasive tubular cancer & $9(3.8)$ & $2(1.0)$ & $31(7.7)$ & $3(3.7)$ & $2(6.3)$ & $47(5.0)$ \\
\hline Invasive mucinous cancer & $13(5.4)$ & $2(1.0)$ & $1(0.3)$ & $1(1.3)$ & $0(0)$ & $17(1.8)$ \\
\hline Other & $22(9.2)$ & $4(2.1)$ & $8(1.9)$ & $1(1.3)$ & $0(0)$ & $35(3.6)$ \\
\hline Missing & 27 & 9 & 12 & 3 & 9 & 60 \\
\hline \multicolumn{7}{|l|}{ Oestrogen receptor } \\
\hline Negative & $42(18.4)$ & $25(13.9)$ & $16(4.1)$ & $17(25.8)$ & $2(6.2)$ & $102(11.4)$ \\
\hline Positive & $186(81.6)$ & $155(86.1)$ & $372(95.9)$ & $49(74.2)$ & $30(93.8)$ & $792(88.6)$ \\
\hline Missing & 38 & 23 & 28 & 17 & 9 & 115 \\
\hline \multicolumn{7}{|l|}{ Progesterone receptor } \\
\hline Negative & $96(43.0)$ & 77 (44.5) & $115(30.9)$ & $33(51.6)$ & $17(54.8)$ & $338(39.2)$ \\
\hline Positive & $127(57.0)$ & $96(55.5)$ & $257(69.1)$ & $31(48.4)$ & $14(45.2)$ & $525(60.8)$ \\
\hline Missing & 43 & 30 & 44 & 19 & 10 & 146 \\
\hline \multicolumn{7}{|l|}{ HER2 } \\
\hline Negative & $199(90.5)$ & $140(84.9)$ & $342(94.5)$ & $49(84.5)$ & $29(93.6)$ & $759(90.8)$ \\
\hline Positive & $21(9.5)$ & $25(15.1)$ & $20(5.5)$ & $9(15.5)$ & $2(6.4)$ & $77(9.2)$ \\
\hline Missing & 46 & 38 & 54 & 25 & 10 & 173 \\
\hline \multicolumn{7}{|l|}{ Ki67 } \\
\hline Low & $55(27.9)$ & $49(32.5)$ & $146(45.3)$ & $21(37.5)$ & $15(50.0)$ & $286(37.8)$ \\
\hline Intermediate & $67(34.0)$ & $45(29.8)$ & $110(34.2)$ & $16(28.6)$ & $6(20.0)$ & $244(32.3)$ \\
\hline High & $75(38.1)$ & $57(37.7)$ & $66(20.5)$ & $19(33.9)$ & $9(30.0)$ & $226(29.9)$ \\
\hline Missing & 69 & 52 & 94 & 27 & 11 & 253 \\
\hline \multicolumn{7}{|l|}{ Breast cancer subtype } \\
\hline Luminal A-like & $95(46.8)$ & $67(44.1)$ & $215(67.4)$ & $23(45.1)$ & $19(63.3)$ & $419(55.5)$ \\
\hline Luminal B-like & $52(25.6)$ & $43(28.3)$ & $74(23.2)$ & $12(23.5)$ & $7(23.3)$ & $188(24.9)$ \\
\hline HER2+ subtype & $21(10.3)$ & $25(16.5)$ & $20(6.3)$ & $9(17.7)$ & $2(6.7)$ & $77(10.2)$ \\
\hline Triple-negative breast cancer & $35(17.2)$ & $17(11.1)$ & $10(3.1)$ & $7(13.7)$ & $2(6.7)$ & $71(9.4)$ \\
\hline Missing & 63 & 51 & 97 & 32 & 11 & 254 \\
\hline
\end{tabular}

Table 1. Patient and tumour characteristics in relation to mammographic appearance. Data presented as counts (percent) unless otherwise stated. 


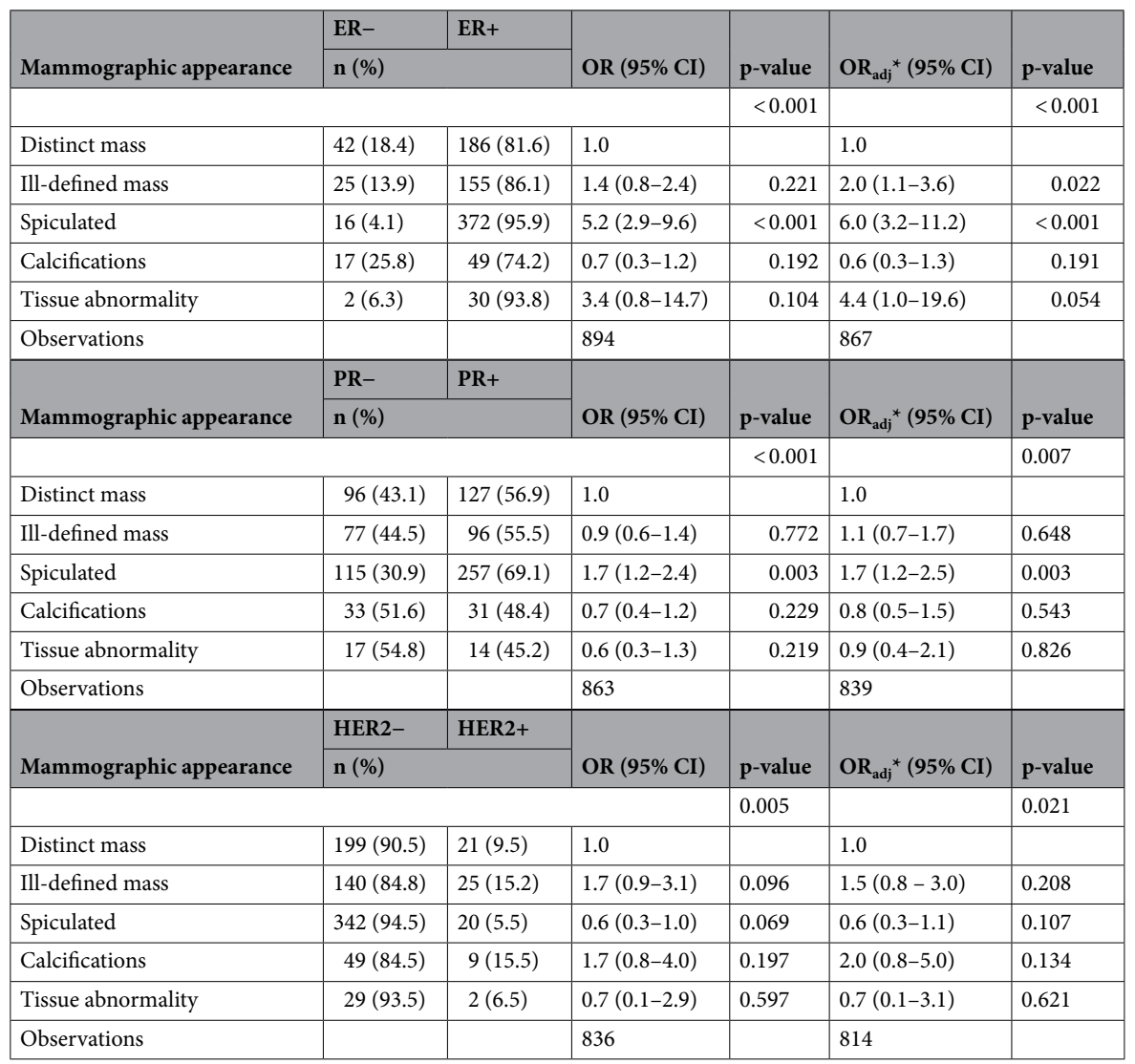

Table 2. Mammographic appearance in relation to oestrogen receptor (ER), progesterone receptor (PR) and human epidermal growth factor 2 (HER2) status. ${ }^{\star}$ Adjusted for age (categorical), tumour size, mode of detection and breast density.

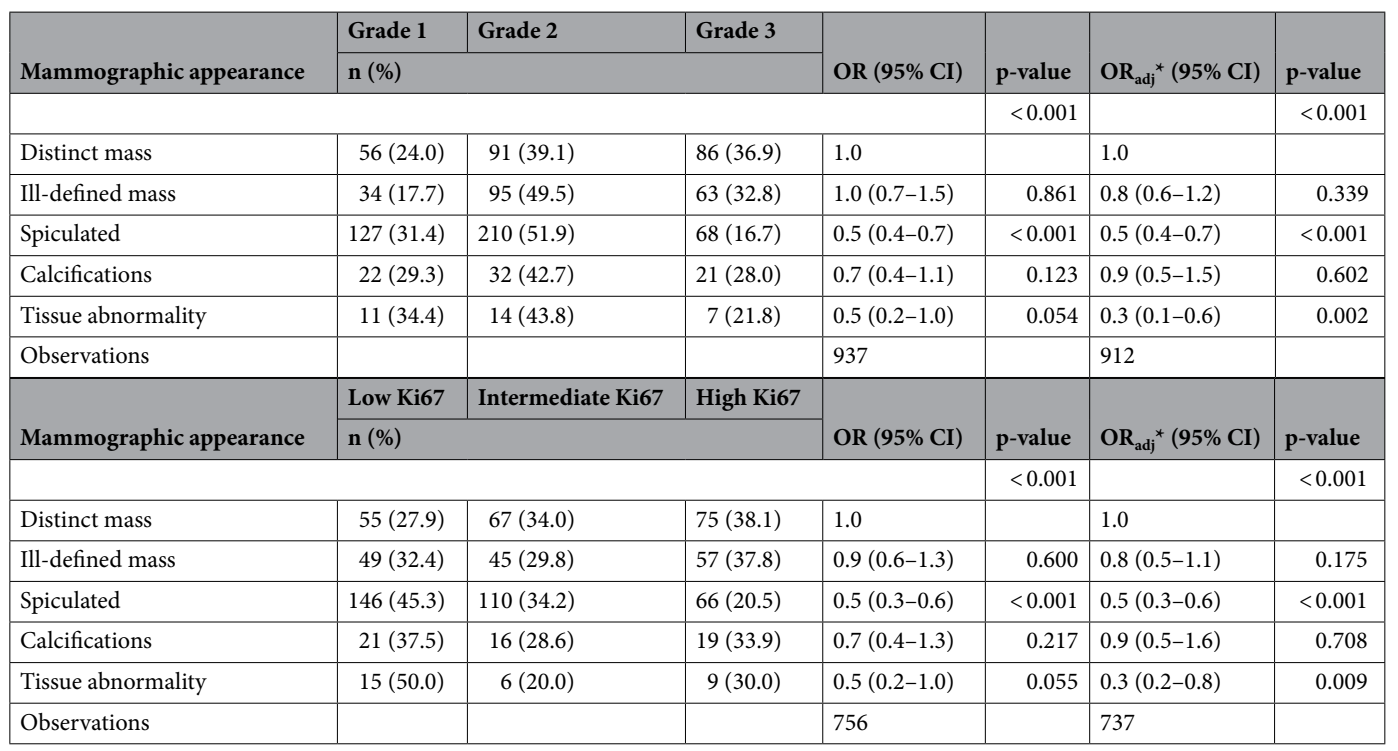

Table 3. Mammographic appearance in relation to histological grade and Ki67 expression. ${ }^{\star}$ Adjusted for age (categorical), tumour size, mode of detection and breast density.

Tumours presenting as tissue abnormalities were also more likely to be of grade 1 or 2 , rather than grade 3 , compared to distinct masses, with an $\mathrm{OR}_{\mathrm{adj}}$ of 0.3 (CI 0.1-0.6). Spiculated tumours were more likely to exhibit lower $\mathrm{Ki} 67$ expression compared to distinct masses, with an $\mathrm{OR}_{\text {adj }}$ of high Ki67 compared to the combined categories 


\begin{tabular}{|l|c|l|l|l|}
\hline Molecular subtype & Luminal A-like & Luminal B-like & HER2+ subtype & Triple-negative breast cancer \\
\hline Mammographic appearance & $\mathbf{n}(\%)$ & \multicolumn{5}{l|}{} \\
\hline Distinct mass & $95(46.8)$ & $52(25.6)$ & $21(10.3)$ & $35(17.3)$ \\
\hline Ill-defined mass & $67(44.1)$ & $43(28.2)$ & $25(16.5)$ & $17(11.2)$ \\
\hline Spiculated & $215(67.4)$ & $74(23.2)$ & $20(6.3)$ & $10(3.1)$ \\
\hline Calcifications & $23(45.1)$ & $12(23.5)$ & $9(17.7)$ & $7(13.7)$ \\
\hline Tissue abnormality & $19(63.3)$ & $7(23.3)$ & $2(6.7)$ & $2(6.7)$ \\
\hline
\end{tabular}

Table 4. Mammographic appearance and frequencies of molecular subtypes.

\begin{tabular}{|c|c|c|c|c|c|c|c|}
\hline \multirow[b]{2}{*}{ Molecular subtype } & \multirow{2}{*}{$\begin{array}{l}\text { Luminal A-like } \\
\text { Reference }\end{array}$} & \multirow{2}{*}{\begin{tabular}{|l|} 
Luminal B-like \\
RRR (95\% CI) \\
\end{tabular}} & \multirow[b]{2}{*}{ p-value } & \multirow{2}{*}{$\begin{array}{l}\begin{array}{l}\text { Human epidermal } \\
\text { growth factor } 2 \\
\text { (HER2)+ subtype }\end{array} \\
\text { RRR (95\% CI) }\end{array}$} & \multirow[b]{2}{*}{ p-value } & \multirow{2}{*}{$\begin{array}{l}\begin{array}{l}\text { Triple negative } \\
\text { breast cancer }\end{array} \\
\text { RRR }(95 \% \mathrm{CI})\end{array}$} & \multirow[b]{2}{*}{ p-value } \\
\hline & & & & & & & \\
\hline \multicolumn{7}{|c|}{ Mammographic appearance } & $<0.001$ \\
\hline Distinct mass & & 1.0 & & 1.0 & & 1.0 & \\
\hline Ill-defined mass & & $1.2(0.7-2.0)$ & 0.541 & $1.7(0.9-3.3)$ & 0.119 & $0.7(0.4-1.3)$ & 0.267 \\
\hline Spiculated & & $0.6(0.4-1.0)$ & 0.034 & $0.4(0.2-0.8)$ & 0.010 & $0.1(0.1-0.3)$ & $<0.001$ \\
\hline Calcifications & & $1.0(0.4-2.1)$ & 0.904 & $1.8(0.7-4.4)$ & 0.216 & $0.8(0.3-2.1)$ & 0.687 \\
\hline Tissue abnormality & & $0.7(0.3-1.7)$ & 0.404 & $0.5(0.1-2.2)$ & 0.342 & $0.3(0.1-1.3)$ & 0.342 \\
\hline Observations & & \multicolumn{6}{|l|}{755} \\
\hline \multirow[b]{2}{*}{ Molecular subtype } & Luminal A-like & Luminal B-like & \multirow[b]{2}{*}{ p-value } & HER2+ subtype & \multirow[b]{2}{*}{ p-value } & \begin{tabular}{|l|}
$\begin{array}{l}\text { Triple negative } \\
\text { breast cancer }\end{array}$ \\
\end{tabular} & \multirow[b]{2}{*}{ p-value } \\
\hline & Reference & $\operatorname{RRR}_{\text {adj }}{ }^{*}(95 \% \mathrm{CI})$ & & $\operatorname{RRR}_{\text {adj }}{ }^{*}(95 \% \mathrm{CI})$ & & $\operatorname{RRR}_{\text {adj }}{ }^{*}(95 \% \mathrm{CI})$ & \\
\hline \multicolumn{7}{|c|}{ Mammographic appearance } & $<0.001$ \\
\hline Distinct mass & & 1.0 & & 1.0 & & 1.0 & \\
\hline Ill-defined mass & & $0.9(0.5-1.6)$ & 0.844 & $1.4(0.7-2.8)$ & 0.387 & $0.5(0.2-0.9)$ & 0.033 \\
\hline Spiculated & & $0.6(0.4-1.0)$ & 0.061 & $0.4(0.2-0.8)$ & 0.015 & $0.1(0.1-0.3)$ & $<0.001$ \\
\hline Calcifications & & $1.4(0.6-3.1)$ & 0.464 & $2.4(0.9-6.5)$ & 0.085 & $0.9(0.3-2.5)$ & 0.861 \\
\hline Tissue abnormality & & $0.4(0.1-1.1)$ & 0.069 & $0.4(0.1-1.7)$ & 0.197 & $0.2(0.0-0.8)$ & 0.023 \\
\hline Observations & & \multicolumn{6}{|l|}{737} \\
\hline
\end{tabular}

Table 5. Mammographic appearance in relation to molecular subtypes. ${ }^{\star}$ Adjusted for age (categorical), tumour size, mode of detection and breast density.

of low and intermediate Ki67 of 0.5 (CI 0.3-0.6). In addition, tissue abnormality had lower odds of being in the high Ki67 expression category as compared to distinct masses, with an $\mathrm{OR}_{\text {adj }}$ of 0.3 (CI 0.2-0.8) (Table 3).

Tumour appearance in relation to surrogate molecular subtypes. The frequencies of the mammographic appearances within the four breast cancer subtypes are given in Table 4. It was found to be more likely that an ill-defined mass would be of luminal A-like subtype than TNBC, than would a distinct mass, with an $\mathrm{RRR}_{\mathrm{adj}}$ of 0.5 (CI 0.2-0.9), according to multinomial logistic regression analysis (Table 5). The relative risk of a spiculated tumour, compared to a distinct mass, being luminal B-like, HER2+ subtype or TNBC, compared to luminal A-like was lower: $\mathrm{RRR}_{\text {adj }} 0.6$ (CI 0.4-1.0), 0.4 (CI 0.2-0.8) and $0.1(0.1-0.3)$, respectively. Or expressed more generally, it is more likely that a spiculated tumour will be luminal A-like than luminal B-like, HER2+ subtype or TNBC. No evidence was found of any association between molecular subtype and tumours presenting as calcifications. Moreover, tumours presenting as tissue abnormality were less likely to be TNBC than luminal A-like, with an $\mathrm{RRR}_{\mathrm{adj}}$ of 0.2 (CI 0.0-0.8) (Table 5).

\section{Discussion}

In this large study on 1116 cases of incident breast cancer, we found strong evidence supporting that established clinicopathological factors and surrogate molecular subtypes differ in regard to mammographic tumour appearance. A particularly interesting result was that all the methods of statistical analysis indicated an association between spiculation and favourable tumour characteristics.

The finding that spiculated tumours are more often ER+ and $\mathrm{PR}+$ is in agreement with several previous studies $^{23-27}$. However, the cut-off values for hormone receptor positivity and tumour appearance categorization differ between some studies ${ }^{23,26}$. In agreement with several other studies, we found spiculation to be associated with lower histological grade ${ }^{28-30}$ and lower Ki67 values ${ }^{23,26}$. A previous study ${ }^{31}$, in which a different categorization of mammographic appearance was used, revealed an association between higher histological grade and spiculation with calcifications. However, in that study, the association with higher histological grade was even 
stronger in non-spiculated tumours, both with and without calcifications. Despite some methodological differences between our study and previous studies, it is clear that spiculation is indicative of favourable tumour characteristics. On the cellular level, the spicules of malignant tumours can represent tumour infiltration, a desmoplastic response in the adjacent stroma or periductal fibrosis ${ }^{32}$. However, the link between these features and the favourable characteristics of spiculated tumours is not clear. Future studies on spiculation and the molecular mammary microenvironment may increase this knowledge.

According to our findings, ill-defined masses were also more likely to be ER+, however, we could find no evidence in the literature supporting or contradicting this finding. This could be because the category ill-defined mass is seldom used, and when it is, often with other outcomes ${ }^{13,29,33}$ than those considered in the present study. Regarding HER2, we found no evidence of any association with tumour appearance. Previous studies have shown an association between spiculation and HER2 $-{ }^{23,26}$ as well as calcifications and HER2 $+{ }^{9,34-36}$. In terms of frequency, the majority of spiculated tumours in our study were HER2- (94.5\%), and calcifications were more often HER2+(15.5\%). Hence, our results point in the same direction, although with weak statistical support. Tumours presenting as tissue abnormality are more likely to be ER+, of lower histologic grade and lower Ki67 expression. The number of observations in this group is however small, making it difficult to draw any reliable conclusions.

Information on surrogate molecular subtypes is essential in breast cancer management. These were therefore included in this study to refine our analyses and to make the results more clinically comprehensible. Spiculated tumours were more commonly luminal A-like subtype, which is in line with the findings of three previous studies $^{26,33,37}$, one of which ${ }^{37}$ highlighted women under the age of 40 . Breast density, which is higher in younger women, is an interesting factor that can affect tumour appearance to some degree. By adjusting for breast density, we were able to show that the association between spiculation and luminal A-like subtype persists, regardless of density. Tamaki et al. ${ }^{38}$ found that masses classified as having indistinct margins on mammography, according to BI-RADS, were more often HER2 + subtype or TNBC than luminal type cancers. This is in contrast to our finding that ill-defined masses are more often luminal A-like than TNBC. The reason for this discrepancy is not known, but could be due to differences in mammographic categorization and population sampling. In the present study, the appearance was extracted from the original radiology report, and BI-RADS is not used in clinical practice at our department. In addition, their population was younger, with a median age of 50 years (range 27-89 years), compared to a median age of 66 years (range 45-91 years) in the present study. Associations between various types of calcifications and HER+ subtype have been described previously ${ }^{36,39,40}$. However, we found no evidence of this in our study. In order to increase group sizes, we combined all types of calcifications into one group, and it was therefore not possible to study differences between various types of calcifications. Several other studies have reported that TNBC is associated with a mammographic mass and can be mistaken for a benign breast lesion ${ }^{10,41-44}$. It is therefore of the utmost importance to investigate these lesions carefully. In terms of frequency, a large proportion of the distinct masses in our study constituted TNBC (17.3\%), as opposed to spiculated tumours, of which only a few (3.1\%) were TNBC (Table 4 ). However, a potential association between TNBC and distinct mass in our material cannot be directly identified due to the setup of statistical analysis.

Some issues require consideration. Firstly, this was a retrospective observational study performed at a single department. Secondly, the women with breast cancer in the MDCS tend to be of ethnic Swedish descent, and with a higher level of education than the average population ${ }^{45}$, which could limit the representativeness of the findings. However, the clinicopathological factors studied were distributed as expected in routine clinical practice. Hence, we believe there is a low risk of selection bias, and that the internal comparisons should not be affected. Thirdly, the use of TMA for evaluation of clinicopathological parameters is worth considering as there is always a risk that the cores obtained for TMA do not reflect the original tumour and its potential heterogeneity correctly. However, this risk is reduced by obtaining two cores from different areas of each tumour, an approach that has been shown to be highly representative in breast $\operatorname{cancer}^{46}$. Furthermore, agreement between TMA assessment and clinical records has been shown to be high ${ }^{47}$. Fourthly, digital mammography was implemented in 2004, and therefore both analogue and digital images were included in this study. However, this has been shown not to influence screening performance ${ }^{48}$. Finally, a wide range of mammographic appearances is used in the literature ${ }^{6,10,26}$, which leads to difficulties when comparing studies. Also, we only considered the most dominant appearance, while others have considered combinations of appearances. Nevertheless, we believe our results to be generalizable, as spiculated appearance is often treated as a separate entity in the literature. This study confirms the clinical notion that breast tumours with a spiculated appearance on mammography have more favourable characteristics and hence the prognosis is potentially better. Survival was not an endpoint in this study, but is planned for a future study. Overdiagnosis of slow-growing tumours that would perhaps not have led to breast cancer morbidity or mortality is a well-known issue in breast cancer screening. In this study, spiculated tumours were more frequent among screening-detected than clinically detected tumours, which may indicate that some of these tumours were so-called over-diagnosed cancers.

In conclusion, this study provides strong statistical evidence of several associations between mammographic tumour appearance and clinicopathological factors, including molecular subtypes. In particular, the results consistently indicate favourable characteristics of spiculated tumours. Defining associations between the mammographic tumour appearance and the clinicopathological outcome may aid in characterizing breast cancer already from the initial mammogram, which could potentially contribute to more individualized breast cancer treatment in the future.

\section{Data availability}

The data supporting the findings of this study are available from the Malmö Cohorts. However, restrictions apply to the availability of the data, which were used under license for the current study, and are not publicly available. For more information visit the Malmö Cohorts webpage: https://www.malmo-kohorter.lu.se/English. 
Received: 15 April 2020; Accepted: 5 November 2020

Published online: 30 November 2020

\section{References}

1. Eccles, S. A. et al. Critical research gaps and translational priorities for the successful prevention and treatment of breast cancer. Breast Cancer Res. 15, R92. https://doi.org/10.1186/bcr3493 (2013).

2. Cherel, P., Becette, V. \& Hagay, C. Stellate images: Anatomic and radiologic correlations. Eur. J. Radiol. 54, 37-54. https://doi. org/10.1016/j.ejrad.2004.11.018 (2005).

3. Berment, H., Becette, V., Mohallem, M., Ferreira, F. \& Cherel, P. Masses in mammography: What are the underlying anatomopathological lesions?. Diagn. Interv. Imaging 95, 124-133. https://doi.org/10.1016/j.diii.2013.12.010 (2014).

4. Tabar, L. et al. Mammographic tumor features can predict long-term outcomes reliably in women with 1-14-mm invasive breast carcinoma. Cancer 101, 1745-1759. https://doi.org/10.1002/cncr.20582 (2004).

5. Sartor, H. et al. Do mammographic tumor features in breast cancer relate to breast density and invasiveness, tumor size, and axillary lymph node involvement?. Acta Radiol. (Stockholm, Sweden) 56, 536-544. https://doi.org/10.1177/0284185114532081 (2015).

6. Alexander, M. C., Yankaskas, B. C. \& Biesemier, K. W. Association of stellate mammographic pattern with survival in small invasive breast tumors. Am. J. Roentgenol. 187, 29-37. https://doi.org/10.2214/ajr.04.0582 (2006).

7. Andersson, I. et al. Breast tomosynthesis and digital mammography: A comparison of breast cancer visibility and BIRADS classification in a population of cancers with subtle mammographic findings. Eur. Radiol. 18, 2817-2825. https://doi.org/10.1007/ s00330-008-1076-9 (2008).

8. Curigliano, G. et al. De-escalating and escalating treatments for early-stage breast cancer: The St. Gallen International Expert Consensus Conference on the Primary Therapy of Early Breast Cancer 2017. Ann. Oncol. 28, 1700-1712. https://doi.org/10.1093/ annonc/mdx308 (2017).

9. Radenkovic, S. et al. HER2-positive breast cancer patients: Correlation between mammographic and pathological findings. Radiat. Prot. Dosimetry 162, 125-128. https://doi.org/10.1093/rpd/ncu243 (2014).

10. Killelea, B. K. et al. Is there a correlation between breast cancer molecular subtype using receptors as surrogates and mammographic appearance?. Ann. Surg. Oncol. 20, 3247-3253. https://doi.org/10.1245/s10434-013-3155-7 (2013).

11. Manjer, J., Elmstahl, S., Janzon, L. \& Berglund, G. Invitation to a population-based cohort study: Differences between subjects recruited using various strategies. Scand. J. Public Health 30, 103-112. https://doi.org/10.1080/14034940210133771 (2002).

12. Berglund, G., Elmstahl, S., Janzon, L. \& Larsson, S. A. The Malmo Diet and Cancer Study. Design and feasibility. J. Internal Med. 233, 45-51 (1993).

13. Luck, A. A. et al. Breast carcinoma with basal phenotype: Mammographic findings. Am. J. Roentgenol. 191, 346-351. https://doi. org/10.2214/ajr.07.2659 (2008).

14. Sickles, E. et al. ACR BI-RADS ${ }^{\star}$ Atlas, Breast Imaging Reporting and Data System (American College of Radiology, New York, 2013).

15. Elebro, K., Butt, S., Dorkhan, M., Jernstrom, H. \& Borgquist, S. Age at first childbirth and oral contraceptive use are associated with risk of androgen receptor-negative breast cancer: The Malmo Diet and Cancer Cohort. Cancer Causes Control 25, 945-957. https://doi.org/10.1007/s10552-014-0394-2 (2014).

16. Dowsett, M. et al. Correlation between immunohistochemistry (HercepTest) and fluorescence in situ hybridization (FISH) for HER-2 in 426 breast carcinomas from 37 centres. J. Pathol. 199, 418-423. https://doi.org/10.1002/path.1313 (2003).

17. Elston, C. W. \& Ellis, I. O. Pathological prognostic factors in breast cancer. I. The value of histological grade in breast cancer: Experience from a large study with long-term follow-up. Histopathology 19, 403-410 (1991).

18. Huss, L. et al. Vitamin D receptor expression in invasive breast tumors and breast cancer survival. Breast Cancer Res. 21, 84. https ://doi.org/10.1186/s13058-019-1169-1 (2019).

19. Dowsett, M. et al. Assessment of Ki67 in breast cancer: Recommendations from the International Ki67 in Breast Cancer working group. J. Natl. Cancer Inst. 103, 1656-1664. https://doi.org/10.1093/jnci/djr393 (2011).

20. Focke, C. M. et al. Interlaboratory variability of Ki67 staining in breast cancer. Eur. J. Cancer (Oxford, England: 1990) 84, 219-227. https://doi.org/10.1016/j.ejca.2017.07.041 (2017).

21. Goldhirsch, A. et al. Personalizing the treatment of women with early breast cancer: Highlights of the St Gallen International Expert Consensus on the Primary Therapy of Early Breast Cancer 2013. Ann. Oncol. 24, 2206-2223. https://doi.org/10.1093/annon c/mdt303 (2013)

22. Guidelines for surgical and oncologic treatment of breast cancer-regional adaption of the national treatment guidelines, Region West and South (in Swedish). https://www.cancercentrum.se/globalassets/cancerdiagnoser/brost/syd/sydsvenska-brostcancergrup pens-lathund-170401-180331.pdf (2017).

23. Jiang, L. et al. Mammographic features are associated with clinicopathological characteristics in invasive breast cancer. Anticancer Res. 31, 2327-2334 (2011).

24. Broberg, A., Glas, U., Gustafsson, S. A., Hellstrom, L. \& Somell, A. Relationship between mammographic pattern and estrogen receptor content in breast cancer. Breast Cancer Res. Treat. 3, 201-207. https://doi.org/10.1007/bf01803562 (1983).

25. Ildefonso, C. et al. The mammographic appearance of breast carcinomas of invasive ductal type: Relationship with clinicopathological parameters, biological features and prognosis. Eur. J. Obstet. Gynecol. Reprod. Biol. 136, 224-231. https://doi.org/10.1016/j. ejogrb.2006.10.025 (2008).

26. Liu, S. et al. Is there a correlation between the presence of a spiculated mass on mammogram and luminal A subtype breast cancer?. Korean J. Radiol. 17, 846-852. https://doi.org/10.3348/kjr.2016.17.6.846 (2016).

27. Nielsen, N. S. \& Poulsen, H. S. Relation between mammographic findings and hormonal receptor content in breast cancer. Am. J. Roentgenol. 145, 501-504. https://doi.org/10.2214/ajr.145.3.501 (1985).

28. De Nunzio, M. C. et al. Correlations between the mammographic features of screen detected invasive breast cancer and pathological prognostic factors. Breast 6, 146-149. https://doi.org/10.1016/S0960-9776(97)90556-7 (1997).

29. Evans, A. J., Pinder, S. E., James, J. J., Ellis, I. O. \& Cornford, E. Is mammographic spiculation an independent, good prognostic factor in screening-detected invasive breast cancer?. Am. J. Roentgenol. 187, 1377-1380. https://doi.org/10.2214/ajr.05.0725 (2006).

30. Lamb, P. M., Perry, N. M., Vinnicombe, S. J. \& Wells, C. A. Correlation between ultrasound characteristics, mammographic findings and histological grade in patients with invasive ductal carcinoma of the breast. Clin. Radiol. 55, 40-44. https://doi.org/10.1053/ crad.1999.0333 (2000).

31. Shin, H. J. et al. Correlation between mammographic and sonographic findings and prognostic factors in patients with nodenegative invasive breast cancer. Br. J. Radiol. 84, 19-30. https://doi.org/10.1259/bjr/92960562 (2011).

32. Franquet, T., De Miguel, C., Cozcolluela, R. \& Donoso, L. Spiculated lesions of the breast: Mammographic-pathologic correlation. Radiogr. Rev. Publ. Radiol. Soc. N. Am. Inc 13, 841-852. https://doi.org/10.1148/radiographics.13.4.8356272 (1993).

33. Taneja, S. et al. The mammographic correlations of a new immunohistochemical classification of invasive breast cancer. Clin. Radiol. 63, 1228-1235. https://doi.org/10.1016/j.crad.2008.06.006 (2008).

34. Gajdos, C. et al. Mammographic appearance of nonpalpable breast cancer reflects pathologic characteristics. Ann. Surg. 235, 246-251 (2002).

35. Sun, S. S., Zhang, B., Zhao, H. M. \& Cao, X. C. Association between mammographic features and clinicopathological characteristics in invasive ductal carcinoma of breast cancer. Mol. Clin. Oncol. 2, 623-629. https://doi.org/10.3892/mco.2014.297 (2014). 
36. Elias, S. G. et al. Imaging features of HER2 overexpression in breast cancer: A systematic review and meta-analysis. Cancer epidemiol. Biomark. Prev. 23, 1464-1483. https://doi.org/10.1158/1055-9965.Epi-13-1170 (2014).

37. Bullier, B. et al. Imaging features of sporadic breast cancer in women under 40 years old: 97 cases. Eur. Radiol. 23, 3237-3245. https://doi.org/10.1007/s00330-013-2966-z (2013).

38. Tamaki, K. et al. Correlation between mammographic findings and corresponding histopathology: Potential predictors for biological characteristics of breast diseases. Cancer Sci. 102, 2179-2185. https://doi.org/10.1111/j.1349-7006.2011.02088.x (2011).

39. Bare, M. et al. Mammographic and clinical characteristics of different phenotypes of screen-detected and interval breast cancers in a nationwide screening program. Breast Cancer Res. Treat. 154, 403-415. https://doi.org/10.1007/s10549-015-3623-9 (2015).

40. Cen, D. et al. BI-RADS 3-5 microcalcifications can preoperatively predict breast cancer HER2 and Luminal a molecular subtype. Oncotarget 8, 13855-13862. https://doi.org/10.18632/oncotarget.14655 (2017).

41. Boisserie-Lacroix, M. et al. Triple-negative breast cancers: Associations between imaging and pathological findings for triplenegative tumors compared with hormone receptor-positive/human epidermal growth factor receptor-2-negative breast cancers. Oncologist 18, 802-811. https://doi.org/10.1634/theoncologist.2013-0380 (2013).

42. Dogan, B. E. \& Turnbull, L. W. Imaging of triple-negative breast cancer. Ann. Oncol. 23(Suppl 6), 23-29. https://doi.org/10.1093/ annonc/mds191 (2012).

43. Gao, B. et al. Mammographic and clinicopathological features of triple-negative breast cancer. Br. J. Radiol. 87, 20130496. https:// doi.org/10.1259/bjr.20130496 (2014).

44. Kim, M. Y. \& Choi, N. Mammographic and ultrasonographic features of triple-negative breast cancer: A comparison with other breast cancer subtypes. Acta Radiol. (Stockholm, Sweden: 1987) 54, 889-894. https://doi.org/10.1177/0284185113488580 (2013).

45. Manjer, J. et al. The Malmo Diet and Cancer Study: Representativity, cancer incidence and mortality in participants and nonparticipants. Eur. J. Cancer prev. 10, 489-499 (2001).

46. Camp, R. L., Charette, L. A. \& Rimm, D. L. Validation of tissue microarray technology in breast carcinoma. Lab. Investig. J. Tech. Methods Pathol. 80, 1943-1949. https://doi.org/10.1038/labinvest.3780204 (2000).

47. Allott, E. H. et al. Intratumoral heterogeneity as a source of discordance in breast cancer biomarker classification. Breast Cancer Res. 18, 68. https://doi.org/10.1186/s13058-016-0725-1 (2016).

48. Song, S. Y. et al. Comparison of digital and screen-film mammography for breast-cancer screening: A systematic review and metaanalysis. J. Breast Cancer 22, 311-325. https://doi.org/10.4048/jbc.2019.22.e24 (2019).

\section{Acknowledgements}

The authors would like to thank Anna Hwasser for data management, and Anna Åkesson for statistical support.

\section{Author contributions}

Conception and design: L.S., H.S., S.Z. Data acquisition: L.S., H.S., S.Z. Data analysis: L.S., H.S. Data interpretation: all authors. Manuscript writing: L.S., H.S. Revision of manuscript: all authors. Final approval of manuscript: all authors.

\section{Funding}

Open Access funding provided by Lund University.

\section{Competing interests}

The authors declare no competing interests.

\section{Additional information}

Correspondence and requests for materials should be addressed to L.S.

Reprints and permissions information is available at www.nature.com/reprints.

Publisher's note Springer Nature remains neutral with regard to jurisdictional claims in published maps and institutional affiliations.

(c) (i) Open Access This article is licensed under a Creative Commons Attribution 4.0 International cc) License, which permits use, sharing, adaptation, distribution and reproduction in any medium or format, as long as you give appropriate credit to the original author(s) and the source, provide a link to the Creative Commons licence, and indicate if changes were made. The images or other third party material in this article are included in the article's Creative Commons licence, unless indicated otherwise in a credit line to the material. If material is not included in the article's Creative Commons licence and your intended use is not permitted by statutory regulation or exceeds the permitted use, you will need to obtain permission directly from the copyright holder. To view a copy of this licence, visit http://creativecommons.org/licenses/by/4.0/.

(C) The Author(s) 2020 